\title{
Retour sur l'évolution universitaire en France
}

Review on the Evolution of Universities in France

Julien Duval

\section{(2) OpenEdition}

Journals

Édition électronique

URL : http://journals.openedition.org/questionsdecommunication/8423

DOI : 10.4000 /questionsdecommunication. 8423

ISSN : 2259-8901

\section{Éditeur}

Presses universitaires de Lorraine

\section{Édition imprimée}

Date de publication : 31 août 2013

Pagination : 211-230

ISBN : 978-2-8143-0162-7

ISSN : 1633-5961

\section{Référence électronique}

Julien Duval, « Retour sur l'évolution universitaire en France», Questions de communication [En ligne], 23 | 2013, mis en ligne le 31 août 2015, consulté le 20 avril 2019. URL : http://journals.openedition.org/ questionsdecommunication/8423; DOI : 10.4000/questionsdecommunication.8423 


\title{
$>$ ÉCHANGES
}

JULIEN DUVAL

Centre européen de sociologie et de science politique

Centre national de la recherche scientifique

F-75013

jduval@msh-paris.fr

\section{RETOUR SUR L'ÉVOLUTION UNIVERSITAIRE EN FRANCE}

\begin{abstract}
Résumé. - Cet article se propose d'apporter quelques éléments complémentaires à l'analyse d'Arnaud Mercier (20 I2). Pour ne pas trop reproduire les oppositions simplistes des discours des réformateurs, la critique des réformes actuelles gagne à tenir compte de caractéristiques en quelque sorte historiques de l'université : les fonctions de l'Université sont un enjeu de luttes, à l'intérieur et à l'extérieur de celle-ci ; et le monde académique est diversifié. Dans cette perspective, les réformes actuelles sont lues comme un signe du rôle croissant que le monde économique joue aujourd'hui dans la définition des « missions » de l'Université. Elles apparaissent également comme participant d'un programme politique plus large qui touche de nombreux domaines de l'activité sociale et l'on est conduit à s'interroger sur les différentes implications que les réformes recouvrent selon les disciplines.
\end{abstract}

Mots clés. - Réformes, universités, recherche, économie, autonomie, communauté universitaire. 
$\mathrm{P}$ our analyser les effets des réformes en cours dans l'enseignement supérieur et la recherche, Arnaud Mercier (20/2) mobilise les outils des sciences sociales. Cette démarche est partagée avec un nombre relativement important d'articles et de livres parus ces dernières années, dont, d'ailleurs, l'auteur propose une utile synthèse. Le texte fait aussi œuvre de témoignage, notamment sur les nouvelles conditions de travail, la démobilisation et le découragement des enseignants-chercheurs. II fournit un contre-argumentaire riche qui tend à renverser les arguments des réformateurs. Les réformes se révèlent une entreprise de destruction dont les principales victimes sont « les valeurs universitaires » (ibid. : 20l), « les missions mêmes de l'institution » (ibid. : 199), ses « priorités originelles » (ibid. : 223) ou encore «l'essence même de la création des universités » (ibid. : 228). Arnaud Mercier aide à ré-envisager sous un jour positif une conception traditionnelle de l'activité académique - ou le « mode | » de Michael Gibbons et de ses coauteurs (1994). Souvent stigmatisé comme une source d'archaïsmes, le passé, a légué un idéal infiniment précieux.

Efficace dans le cadre d'un « pamphlet académique » (Mercier, 2012 : 200) qui en appelle à « l'esprit de résistance » (ibid. : 230), l'inversion du discours dominant est peut-être moins convaincante dans une perspective strictement analytique. Elle risque d'entretenir les dichotomies chères aux réformateurs et, parfois, l'argumentation peut susciter des réserves, par exemple quand les enseignantschercheurs sont dépeints comme (seulement ?) animés par des « aspirations culturelles et intellectuelles 》 (ibid. : 222), le souci du « bien commun académique », le « goût de l'apprentissage du savoir » (ibid. : 228) ou la volonté de produire et de diffuser « un discours critique sur la société » (ibid. : 229). Les défenseurs des réformes pourraient peut-être retourner au chercheur le reproche de pratiquer une «langue de bois »: les universitaires ne se conforment pas tous, et pas à chaque instant de leur vie professionnelle, à ce portrait idéalisé. Par ailleurs, les « managers » et les universitaires ne forment pas, dans les faits, des groupes étanches et totalement antagonistes ; les premiers sortent même souvent des rangs des seconds. De même, des notions comme les « missions même de l'institution » ou ses « priorités originelles » méritent peut-être des clarifications.

II ne s'agira pas de porter la contradiction à Arnaud Mercier, mais d'esquisser une analyse des réformes qui essaie d'échapper davantage aux oppositions binaires que le discours dominant tend à imposer. Dans un premier temps, quelques éléments historiques sont rassemblés. Ils suggèrent que la question - centrale dans les réformes en cours - de déterminer si les institutions académiques doivent se centrer sur des savoirs relativement fondamentaux et désintéressés, que privilégient leurs segments les plus autonomes, ou sur des savoirs plus immédiatement utiles (et moins critiques), s'est, dans un sens, posée dès les débuts des universités. Le monde académique qui a très tôt été un univers différencié n'y a pas répondu de manière uniforme. Les « missions » de l'université semblent avoir toujours été un enjeu de luttes, ou du moins un objet de désaccords, à l'intérieur et à l'extérieur de l'institution. Dans 
un deuxième temps, le regard porté sur les réformes actuelles mobilise ces considérations. Les réformes, souvent justifiées par le caractère exceptionnel de la période présente (conjoncture de crise, mondialisation, etc.), ne sont en quelque sorte qu'une nouvelle tentative - il y en eut beaucoup par le passé -, par laquelle un pouvoir temporel entreprend de redéfinir à son profit les fonctions de l'Université. Les évolutions en cours gagnent aussi à être rapportées à la diversité très ancienne du monde universitaire, dans la mesure où leurs implications varient selon les disciplines et les segments du monde académique. Enfin, ce texte attire l'attention sur des aspects des réformes - et de la « résistance » (Mercier, 2012 : 204) à laquelle ils peuvent donner lieu peut-être un peu négligés dans la perspective proposée par Arnaud Mercier.

\section{L'autonomie comme compromis}

La référence aux origines de l'Université constituant un argument de poids dans les débats autour de l'enseignement supérieur, il est utile de revenir, même rapidement, sur le moment souvent regardé comme fondateur de la fin du Moyen Âge. Cela peut aider à résister au mythe d'un âge d'or - toujours tentant, comme le remémore Arnaud Mercier (ibid. : 204). De surcroît, la période de la naissance des universités en Europe, et notamment celle de Paris, a l'avantage de faire apparaître, avec la clarté propre aux commencements, des traits de l'institution universitaire qui se dégagent avec moins d'évidence aux époques ultérieures, l'organisation académique ayant gagné en complexité au fil du temps.

\section{Sur les débuts des universités}

Les universités qui se développent en Occident à partir de la fin du XI ${ }^{\mathrm{e}}$ siècle ont participé à un renouveau de la vie intellectuelle'. Pierre Abélard ( |079- | | 42) est l'un des symboles de ces lieux d'innovation intellectuelle qui, comme l'écrit Jacques Le Goff (1999 : 203), contribuent peu à peu « à modifier le caractère de la science et à la détourner de son aspect magique et thésaurisateur pour en faire un savoir rationnel, pratique, communiqué [...] par un apprentissage technique ». Les hommes et les savoirs circulent entre les universités implantées dans différentes villes européennes. Les enseignements sont dispensés gratuitement à un public diversifié par ses origines géographiques mais aussi sociales (noblesse, bourgeoisie, couches aisées de la paysannerie).

\footnotetext{
Sauf indication contraire, dans cette partie, les informations sur les universités au Moyen Âge sont empruntées à Jacques Verger (1973).
} 
D'un point de vue sociologique, ces innovations vont de pair avec la formation et la structuration d'un nouveau groupe social : les maîtres et les étudiants se constituent en une communauté relativement solidaire qui, à Paris, parvient, dès le début du $\mathrm{XII}^{\mathrm{e}}$ siècle, à se faire reconnaître les grandes caractéristiques des corporations du Moyen Âge. Elle se dote de ses propres règles de fonctionnement, aspire à maîtriser son recrutement, s'impose comme une personnalité collective auprès des pouvoirs publics. Le groupe a un fonctionnement assez démocratique : le recteur qui le représente exécute largement les décisions des assemblées de l'université, au sein desquelles les élèves occupent une place parfois centrale.

Mais, les transformations qui se produisent auX $X V^{e}$ et $X V^{e}$ siècles dissuadent de ne voir dans la naissance des universités que l'affirmation d'un idéal intellectuel et l'institutionnalisation de ses conditions sociales d'existence. À Paris, la corporation perd une grande part de l'autonomie qu'elle était parvenue à conquérir, les universités cessent d'être « des centres de travail intellectuel et scientifique désintéressé » pour devenir des sortes de « centres de formation professionnelle au service des États »(Le Goff, 1999 : 207). Entre le XIII et le Xv siècle, les études prennent un caractère utilitaire plus prononcé et les maîtres composent une population plus hétérogène où coexistent, à côté d'un quasi-« prolétariat intellectuel », des universitaires très bien rémunérés comme certains médecins ou juristes réputés. Parmi les élèves, les étudiants riches deviennent plus nombreux. L'ensemble de la vie universitaire connaît un processus d'« aristocratisation » qui a pour envers une raréfaction de l'innovation intellectuelle au profit d'enseignements plus conservateurs et plus routiniers (Verger, 1973 : 105-200).

\section{Une institution ambiguë}

Ces évolutions attirent l'attention sur des ambiguïtés, sans doute constitutives, des universités. II n'est pas possible de réduire le phénomène universitaire au Moyen Âge à l'affirmation d'un pouvoir et d'un idéal intellectuels nouveaux, ou il faut insister sur les compromis que ce pouvoir et cet idéal ont dû passer avec des forces sociales et plusieurs pouvoirs temporels. Comme le soulignait Émile Durkheim (1938: 97-I II), l'autonomie acquise par la corporation universitaire reposait sur des relations particulièrement complexes avec l'Église, consistant notamment à obtenir la protection du pape (et, du même coup, à se placer sous sa dépendance) pour se soustraire au contrôle des pouvoirs religieux locaux. L'autonomie de l'Université par rapport à l'institution religieuse dont elle concurrençait le monopole en matière scolaire, mais dont elle était issue, ne pouvait qu'être partielle.

Loin d'être une institution pure, l'Université s'est constituée en interaction avec des intérêts sociaux qui avaient peu à voir avec un idéal strictement intellectuel. Le Saint-Siège ne la soutenait pas sans la concevoir comme un instrument utile à la centralisation de l'Église et à la lutte contre les hérésies. Quant au pouvoir royal, il était très sensible au prestige que l'université de Paris conférait à la capitale. Peu à 
peu, le roi, les princes ou les municipalités sont intervenus de manière plus active sur le terrain universitaire. II ne s'agissait pas seulement de rétablir l'ordre dans des institutions qui se transformaient régulièrement en foyers d'agitation, mais aussi de les redéfinir de manière à ce qu'elles servent au mieux les intérêts d'administrations qui se développaient, et dont elles formaient un nombre croissant des serviteurs.

Autant qu'un public à la recherche de connaissances ou que des maîtres dévoués à un idéal intellectuel, le pouvoir royal, des municipalités, des seigneurs ou les autorités religieuses ont contribué au développement des nouvelles institutions et aux formes qu'elles ont prises. Les nombreuses universités créées à leur initiative le montrent. Les interventions de ces pouvoirs externes n'allaient pas systématiquement à l'encontre des logiques que les universités naissantes développaient, en fonction des intérêts propres qu'elles se découvraient. Par exemple, Jacques Le Goff ( 1999 : 199) mentionne que « le caractère livresque et théorique » des enseignements de l'époque ne posait pas nécessairement problème aux souverains, alors demandeurs d'un « savoir inspiré par des principes scolastiques ». Cependant, dans certains cas (comme la condamnation en 1270 et en 1277 des thèses averrö̈stes par l'évêque de Paris), les interventions s'apparentaient incontestablement à des rappels à l'ordre et impliquaient des restrictions à la liberté universitaire.

Devant négocier son autonomie, la communauté universitaire était condamnée aux ambiguités communes aux détenteurs de ce pouvoir dominé qu'est le pouvoir intellectuel (Bourdieu, 1989). Devant assurer leur subsistance, les maîtres du Moyen Âge ne pouvaient par exemple réaliser pleinement l'idéal consistant à dispenser gratuitement un savoir pur : compter sur des dons des élèves revenait à renier le principe de gratuité ; et recevoir des revenus de l'Église, d'un prince ou d'une municipalité, fondait ces derniers à demander des contreparties. Les savoirs produits et dispensés se ressentaient des relations de dépendance à l'égard des différents pouvoirs temporels : par exemple, les thèses gallicanes ne pouvaient être défendues qu'avec prudence, l'autonomie de la corporation devant beaucoup au pape. Plus tard, quand elle devient « la fille aînée du roi », l'Université est nécessairement condamnée à une timidité politique (Le Goff, 1999 : 204).

L'ambiguïté de l'Université du Moyen Âge se traduit dans la polysémie, souvent relevée, du terme de « clerc » qui, sill ne désignait à l'origine qu'un homme d'Église, prend un sens de plus en plus large et de plus en plus indéterminé, s'appliquant à celui qui sait le latin, au lettré, mais aussi à un fonctionnaire (Verger, 1973 : 130 ; Le Goff, 1999 : 194). Loin de travailler seulement à sa reproduction, l'Université formait majoritairement des élèves qui, une fois gradués, allaient servir les administrations ecclésiastiques ou étatiques. Émile Durkheim (1938:239) remarquait combien les écoles médiévales avaient contribué à produire des « hommes d'actions » : « c'est là que se formèrent les hommes d'État, les dignitaires ecclésiastiques, les administrateurs de l'époque ».

Des notions tels « l'idéal universitaire » ou « les missions [premières] de l'Université » n'ont donc peut-être jamais été définies de façon claire et univoque. Elles ont vraisemblablement toujours été des enjeux de luttes entre les différents 
groupes sociaux prenant part à l'Université. Dans l'Université médiévale, sans doute des maîtres aspiraient-ils à produire et diffuser un savoir qui, dans des termes modernes, pourrait être qualifié de critique, mais, simultanément, l'Église ou le pouvoir royal assignaient à l'institution des missions partiellement différentes, et parfois même opposées. Par leurs usages de l'Université, les élèves étaient susceptibles de peser sur les missions qu'elle remplissait dans la pratique. De façon générale, chacun percevra comme « première » la fonction de l'Université qu'il privilégie. Et, l'institution semblant s'être caractérisée dès ses débuts par une certaine ambiguïté, chacun pourra se réclamer des « origines » et trouver des arguments « historiques » susceptibles de conforter son point de vue.

\section{La division en facultés}

La corporation universitaire elle-même ne partageait pas une conception unifiée de ses fins. Dès le $X \|^{e}$ siècle, des différences existent parmi les maitres. Aux « affamés de science pure, de la science pour la science », s'opposent (déjà) « ceux qui cherchent seulement à faire commerce de leur savoir » (Le Goff, 1999 : 182). L'organisation en facultés assez autonomes est un signe évident de la diversité du groupe. Trois de ces facultés, le droit, la médecine et la théologie, étaient « comme aujourd'hui des écoles spéciales, professionnelles, qui préparaient à des carrières déterminées », quand la quatrième, les arts libéraux, représentait un « organe de culture générale, désintéressée » (Durkheim, 1938 : | | 8), un « vestibule général » aux trois autres. Ces facultés ne remplissaient pas les mêmes « missions » et n'étaient pas pareillement exposées à l'intervention des pouvoirs externes. La théologie était la plus surveillée par l'Église. Le droit et la médecine avaient vu le jour, sous forme d'écoles, dès les années II 50 et étaient orientés vers la formation de professionnels et, pour le droit, de serviteurs de l'État - et de l'Église (Verger, 1973 :27, 156).

De façon compréhensible, les pouvoirs publics considéraient comme « capital que la faculté dominante soit celle de théologie ou une faculté orientée vers les carrières "lucratives" ou "utilitaires" - droit ou médecine » (Le Goff, 1999 : 193). II en allait peut-être différemment à l'intérieur de la corporation : selon Émile Durkheim (1938: 120), la faculté des arts, qui représentait « une sorte de Faculté inférieure », exerça très tôt, de façon paradoxale, « une véritable hégémonie » sur l'ensemble de la corporation. L'auteur y voyait un signe du rôle fondamental qui, au Moyen Âge, revenait à la dialectique (qu'elle enseignait), lequel renvoyait plus largement, à ses yeux, à une manifestation de l'importance constante que le système d'enseignement français avait accordée à un « formalisme pédagogique » qui, successivement depuis l'école carolingienne, s'était incarné dans la grammaire, la dialectique, puis dans la rhétorique et la mise à distance des « besoins de la vie réelle » dans l'éducation dispensée par les jésuites (ibid. : 265)².

2 La critique par ce fondateur des sciences sociales du « formalisme pédagogique » est intéressante dans le contexte actuel, où les sciences sociales peuvent être tentées d'opposer aux réformes 
La diversité interne de l'Université a traversé le temps. Un moment comme l'affaire Dreyfus révèle qu'il n'y a pas de consensus parmi les universitaires sur la question de savoir si l'Université doit exercer une fonction sociale critique ou, au contraire, soutenir les institutions sociales existantes (Charle, 1994). S'il existe une diversité à l'intérieur des disciplines, la division en facultés reste un facteur de clivage important auX XIX et $X X^{e}$ siècles (Bourdieu, 1984 ; Charle, 1994). Les professeurs de droit et de médecine incarnent un pôle plus « temporel » que les enseignants de lettres et de sciences ${ }^{3}$. Recrutés par l'agrégation, ils forment des corps plus homogènes, et de recrutement social plus élevé. Ils sont moins orientés vers la recherche. Des différences importantes s'observent également entre les lettres et les sciences. Beaucoup plus fréquemment dans les sciences de la nature que dans des disciplines plus littéraires, la recherche prend la forme d'entreprises collectives. Elle y mobilise aussi parfois des moyens matériels importants et peut avoir des retombées économiques (ou militaires). Par ailleurs, du point de vue de l'enseignement, certaines disciplines scientifiques forment, depuis un certain temps, des professionnels pour le secteur privé.

De rapides éléments statistiques sur la population étudiante peuvent compléter ce rappel de la diversité de l'Université. Les filières de lettres et sciences humaines concentrent toujours une forte proportion d'étudiants - 30,4\%, dans une nomenclature qui distingue le droit (13,8\% des effectifs), l'économie et l'administration économique et sociale (AES - 13,1 \%), les sciences et les sciences et techniques des activités physiques et sportives (STAPS - 20,5\%) et la santé (14\%) (MESR, 20I I : 35). La proportion d'enfants de cadres et professions intellectuelles supérieures est plus marquée dans les filières de droit (35,5\%) et de santé (4I,3\%) qu'en sciences (30,7\%) et en lettres (25,5\%) (MEN, 2012 : 195). La part des étudiants de master engagés dans une formation à l'enseignement est plus importante en lettres et arts que dans les autres disciplines et l'enquête sur l'insertion professionnelle des étudiants menée en 2009 confirme que le droit, la santé ou les disciplines scientifiques les plus orientées vers les applications, comme l'informatique, ont les taux d'insertion les plus élevés (MESR, 20 I I :56).

l'idéal d'un savoir pur. Elle rappelle que les sciences sociales se sont en partie construites contre une tradition aristocratique valorisant une culture littéraire esthétique plutôt que des savoirs scientifiques qui « peu[vent] difficilement ne servir à rien du fait qu'[ils] se rapporte[nt] à la réalité » (Durkheim, 1938 :253). Selon une autre formule d'É. Durkheim, les recherches en sciences sociales « ne mériteraient pas une heure de peine si elles ne devaient avoir qu'un intérêt spéculatif ».

${ }^{3}$ Faute de données plus précises, les résultats des élections au Conseil national des universités (CNU) suggèrent que les enseignants sont globalement plus « conservateurs » dans les disciplines des anciennes facultés de droit qu'en lettres et en sciences. Selon les résultats publiés sur le site Galaxie, aux élections de 2011 pour le CNU, la proportion d'élus soutenus par le Syndicat national de l'enseignement supérieur (SNESUP) ou le Syndicat général de l'éducation nationale (SGEN) était chez les professeurs de $8 \%$ dans les disciplines de droit, économie et gestion (groupes I et 2 du CNU), mais de $32 \%$ dans les disciplines scientifiques (groupes 5 à 10) et de $44 \%$ dans les disciplines de lettres et sciences humaines (groupes 3, 4 et 12). Inversement, la proportion d'élus du syndicat autonome était de $26,5 \%$ dans les premières, mais inférieure à $5 \%$ dans les autres. 


\section{Les réformes}

Si « les missions » de l'Université sont, sans doute à toutes les époques, des enjeux de luttes internes et externes à l'institution, une particularité importante de la période présente tient au poids revenant aujourd'hui dans ces luttes à l'économie et, plus précisément, à des organisations et à des groupes qui sont puissants dans le champ économique, tout en disposant de forts relais politiques.

\section{Le changement de conjoncture(s)}

Les justifications en faveur des réformes actuelles du monde académique se réfèrent beaucoup à l'économie. Elles invoquent souvent la dégradation de la conjoncture économique au cours des dernières décennies (ou d'une période plus récente), le ralentissement de la croissance, l'intensification de la concurrence internationale, l'émergence de nouvelles nations économiques, la désindustrialisation, le chômage de masse, etc. Quant au mauvais état des finances publiques, il justifierait des mesures de rationalisation de dépenses et les invitations adressées aux établissements de diversifier leurs sources de financement, en se tournant notamment vers le secteur privé.

Mais, il ne suffit pas d'invoquer la « conjoncture économique » : la représentation qu'on se fait de cette dernière, les orientations politiques qu'on en déduit, l'importance même qui lui est accordée par rapport à d'autres paramètres également susceptibles d'orienter les choix collectifs, sont en partie le produit de rapports de forces politiques. Ces dernières décennies, ceux-ci se sont notablement modifiés et, avec eux, l'humeur idéologique dominante. Le terme « néolibéralisme » est souvent utilisé pour désigner les orientations politiques qui se sont imposées, à un niveau international, d'une façon accélérée après l'arrivée au pouvoir de Ronald Reagan et de Margaret Thatcher. Le néolibéralisme se caractérise par son hostilité à l'État-providence et son soutien aux catégories sociales et entrepreneuriales créatrices de richesses. II marque un retour à une idéologie qui, à la suite d'une éclipse après la guerre, prône de nouveau la subordination de l'ensemble des structures sociales à l'économie (Polanyi, 1944). II s'est notamment imposé dans des instances internationales (Organisation de coopération et de développement économique - OCDE -, Commission européenne...). Son succès est inséparable d'un rapport de forces politiques plus favorable aux organisations dominant le champ économique (notamment, les regroupements d'intérêts patronaux, comme l'European Table of Industrialists) et qui ont radicalisé leurs positions, et plus défavorable aux représentants des salariés. Le déclin du syndicalisme et la disparition de la menace représentée, à l'après-guerre, par le communisme, sont aussi des aspects de cette transformation idéologique et politique.

Avec les transformations de la conjoncture économique et des rapports de forces politiques, les griefs patronaux relativement anciens à l'égard du système d'enseignement ont eu plus d'écho. En 1975, dans un contexte qui n'était pas le 
même qu'aujourd'hui, Pierre Bourdieu et Luc Boltanski (1975) expliquaient que, en raison de l'accroissement du nombre des diplômés, l'intérêt des « acheteurs de la force de travail » était de réduire l'autonomie relative d'un système scolaire qui dote les étudiants, non pas seulement de « compétences », mais aussi de « titres » pourvus, sur le marché du travail, d'une valeur que les employeurs contrôlent peu. Le « rêve patronal d'une école confondue avec l'entreprise » commençait à se réaliser avec les « écoles-maison », la formation permanente ou les écoles de commerce. De fait, à partir des années 60, la formation permanente s'est développée, le Conseil national du patronat français (CNPF) commençant alors à percevoir et à présenter la formation comme un « impératif industriel » ou un « instrument de rentabilité » et à reprocher à « l'université traditionnelle » de ne pas produire - ou de ne pas le faire dans les bonnes proportions - un personnel doté des qualifications demandées par les grandes entreprises (Bachy, 1975). La « déploration de l'incapacité des diplômés », la critique des savoirs universitaires pour leur caractère abstrait et livresque, constituent des leitmotive patronaux encore plus anciens (Bourdieu, 1989 : 402-404). Les appels contemporains à des réformes de l'enseignement supérieur qui émanent du milieu économique 4 ne sont donc pas simplement dictés par la situation économique ; il s'agit aussi de l'amplification de demandes patronales antérieures aux retournements économiques de ces dernières décennies.

\section{Un programme politique}

Leur donnant plus de force ${ }^{5}$, la conjoncture nouvelle produit des effets dans d'autres domaines de la vie sociale. Les réformes dont le monde académique fait l'objet semblent s'inscrire dans un programme politique plus large (et sans doute d'autant plus efficace dans chaque secteur qu'il est simultanément mis en œuvre dans d'autres domaines, au nom d'arguments très proches).

À cet égard, un parallèle peut être esquissé entre les réformes dans l'univers académique et celles qui ont lieu, par exemple, en matière de protection sociale (Duval, 2007). Dans les deux cas se repèrent la même invocation de la conjoncture économique, le même appel à rationaliser les dépenses en privilégiant les dépenses actives (qui servent l'économie), la même tendance à étouffer le débat autour de ces choix en assimilant toute critique des réformes à une posture économiquement irresponsable. Autres points communs, les réformes sont soutenues par presque les mêmes organisations économiques et leurs promoteurs empruntent

\footnotetext{
${ }^{4}$ On peut noter que les appels contemporains à des réformes de l'enseignement supérieur émanant du milieu économique s'inscrivent dans le cadre de revendications concernant l'ensemble du système éducatif, à partir de l'école primaire (e.g. Parisot, Pébereau, 2012).

${ }^{5}$ La nouvelle conjoncture semble aussi avoir permis une reformulation des demandes : dans les années 70, le patronat ne met pas (encore) en question la « vraie mission » des universités à être « un lieu de culture [...] et d'avancement des connaissances fondamentales de la société » (Bourdieu, Boltanski, 1975).
} 
régulièrement des arguments à l'économie néoclassique. Par exemple, au sujet de l'enseignement supérieur, ils invoquent un lien entre « capital humain » et croissance (ou les bénéfices privés qu'un individu retire d'études supérieures et qui justifieraient un accroissement des droits d'inscription) ou, pour la « nouvelle politique de la recherche », la « théorie du tournoi » (Karpik, 20 I2).

Dans les deux cas s'observe aussi un ensemble d'affirmations spécifiques au domaine qui, bien que fragile quand rigoureusement examiné, a acquis un statut d'évidence dans le débat public. Un peu à la manière du « trou de la sécu », le classement de Shanghai et la mauvaise place qu'y occupent les universités françaises ont joui, en dépit de fortes critiques, d'une visibilité croissante. Depuis sa première édition en 2003, ce n'est pas seulement le bruit médiatique autour du classement qui s'est accru, mais aussi l'attention que lui prêtent des médias généralistes qui, comme la presse quotidienne régionale, en étendent la notoriété auprès d'un large public (figure I). De même que le débat sur la protection sociale s'est s'organisé autour d'un petit nombre de problèmes qui ont fait l'objet d'un travail de communication politique pour légitimer les réformes ${ }^{6}$, la réflexion sur l'enseignement supérieur et la recherche a fait une place écrasante à quelques lieux communs relatifs à des échecs ou à des carences de l'Université et de la recherche : les taux d'abandon en premier cycle, la faible attractivité pour les (« bons ») étudiants étrangers, l'insuffisance des offres « professionnalisantes 》, la « fuite des cerveaux » scientifiques, de mauvaises positions dans des classements internationaux...

Figure I. Nombre d'articles, dans les titres de presse français archivés dans la base Europresse, se rapportant au classement de Shanghaï

(source : J. Duval, d'après la base de données Europresse).

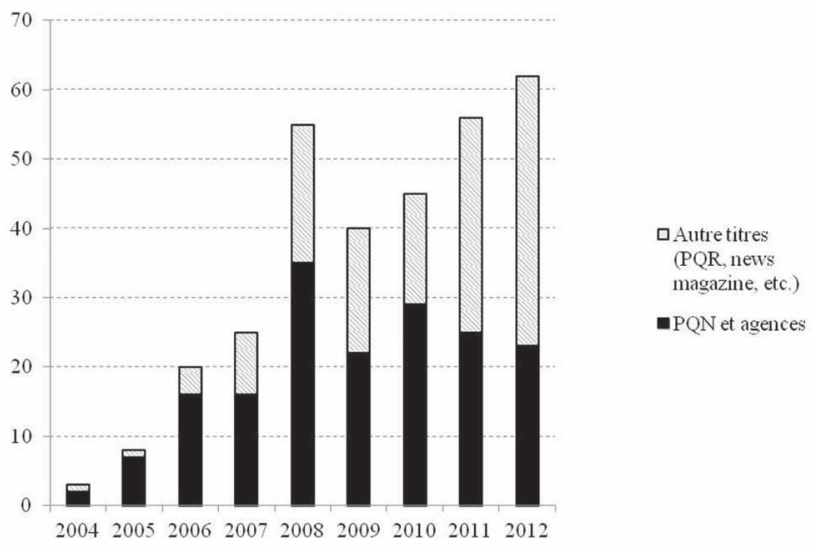

\footnotetext{
6 Si de nombreux travaux de sciences sociales portent sur les réformes en matière d'Université et de recherche, peu utilisent l'approche, couramment mise en œuvre aujourd'hui sur d'autres objets, qui consiste à reconstituer comment des problématiques se constituent et s'installent dans le débat public (e.g. Lenoir, 1989 ; Spector, Kitsuse, 2009).
} 
Un élément de la dynamique actuelle est la redéfinition des activités académiques autour de l'utilité que les groupes dominants dans le monde économique en retirent (en ce sens, parler d'une conception «utilitaire » est trop général).Les réformes tendent à recentrer l'Université (qui ne remplit pas seulement des fonctions économiques, mais aussi culturelles, sociales) sur la fonction que privilégie le champ économique, la formation de salariés dotés des savoirs (les compétences) dont ont besoin, dans un état donné du marché du travail, les entreprises privées. Cette logique conduit à accroître le poids des représentants du monde économique dans les instances universitaires, à réduire la recherche à sa composante dite « pertinente » (c'est-à-dire à la production de résultats générant à court terme des profits pour des entreprises), à mettre en place des « chaires privées », ou des dispositifs fiscaux qui bénéficient aux entreprises investissant dans la recherche-développement universitaire et qui, dans un contexte de rigueur budgétaire, viennent concurrencer les financements publics versés aux universités et aux organismes de recherche. Le monde académique se voit assigner comme fin première de contribuer à la « croissance économique » (de court terme), dans le cadre du mot d'ordre que constitue, depuis le début des années 2000, « l'économie de la connaissance ».

L'« économicisation » de ce monde (Montlibert, 2004 ; Garcia, 2009) consiste à le subordonner à des fins économiques, mais aussi à y introduire des mécanismes propres aux entreprises privées et au «marché ». Les évolutions liées au néolibéralisme passent par l'aura dont est aujourd'hui investi, notamment aux yeux des décideurs publics, ce qui est lié au « marché » et au secteur privé, tandis que ce qui évoque l'État, le service public tend à être, au contraire dévalué et discrédité. Comme nombre de services publics, le monde académique a intégré une vision en termes de « clients » ou de « compétences » (Ropé, Tanguy, 1994), des « démarches qualité », le New Public Management... Les discours réformateurs, comme les transformations des conditions de travail, posent parfois la figure du manager ou du responsable économique en modèle.

Par exemple, les directeurs de laboratoire devraient se convertir en « entrepreneurs académiques », consacrant une partie importante de leur temps à la collecte de fonds, et « manageant », sans exercer de leadership proprement scientifique ou intellectuel, des structures dont les équipes seraient des sortes de filiales, jugées sur leurs « performances ». La tendance des réformateurs à ériger le système universitaire états-unien en un modèle dont la France devrait se rapprocher - en diversifiant les sources de financement des universités, en faisant de ces dernières « le cœur du système de recherche » (Musselin, 2008)... - découle en partie de cette politique d'économicisation. Aux États-Unis, l'équilibre entre l'initiative privée et l'intervention publique est historiquement spécifique et l'enseignement supérieur y bénéficie, par les fondations et la philanthropie, d'une tradition, quasi inexistante en France, de financements par des sources privées. Par ailleurs, les États-Unis se sont engagés précocement dans les orientations mises en œuvre aujourd'hui dans beaucoup d'autres pays, par exemple en cherchant, dès le début des années 80 , à stimuler « l'innovation » à l'Université (Malissard, Gingras, Gemme, 2003). 
« L'économicisation » recouvre aussi la constitution d'un marché de l'enseignement supérieur et de la recherche. Plutôt que d'être pensées comme les éléments complémentaires d'un système national, les universités ou les unités de recherche tendent alors à l'être comme des organisations (à mettre) en concurrence, afin que les étudiants, les personnels de l'enseignement et de la recherche, les entreprises et les décideurs publics puissent faire les meilleurs « choix ». Les classements en tous genres qui se sont multipliés sont censés éclairer ces agents économiques et rendre le marché «transparent ». Les procédures d'évaluation des unités de recherche, des formations, permettraient aux pouvoirs publics ou à ceux qui attribuent les financements publics de mettre en œuvre une « politique de l'excellence » concentrant les moyens sur les « meilleurs » ou, ex post, de s'assurer, à la manière des actionnaires d'une firme privée, du « retour sur investissement ».

L'étonnement que l'on peut éprouver devant les raisonnements de ce type rappelle ce qu'ils doivent à des points de vue particuliers. L'évaluation, surtout dans le feu de l'action, d'une activité de recherche est difficile. L'histoire intellectuelle et scientifique regorge d'exemples de travaux qui, passés inaperçus en leur temps, se sont avérés dans le long terme beaucoup plus féconds (y compris parfois en termes d'innovations industrielles par exemple) que la production courante, sans doute jugée « meilleure » sur le coup. L'évaluation des apports d'un enseignement ne paraît pas plus simple. L'appréhension du monde universitaire sur le mode du marché est loin d'être générale à tous les agents sociaux : le « choix » d'un lieu d'études, d'une filière ou, pour des enseignants-chercheurs, d'un laboratoire paraît très souvent contraint par des limitations de ressources ou des attaches de toutes sortes (ne serait-ce que familiales). Le « marché » que les réformes entendent construire suppose, au contraire, des individus presqu'aussi mobiles que des capitaux dans un espace financier déréglementé7.

Les réformes ne vont pas sans une dimension conservatrice. Développer les segments de l'activité académique utiles à court terme pour les entreprises a pour contrepartie d'en raréfier d'autres qui pourraient exercer des effets de nuisance. Si, dans l'enseignement supérieur, il n'y a pas eu d'initiative aussi spectaculaire que les révisions dont ont pu faire l'objet, dans l'enseignement secondaire, les programmes de sciences économiques et sociales auxquels il était reproché d'inviter les élèves à porter un regard trop critique sur le monde de l'entreprise, la liberté de la recherche semble avoir pâti des réformes mises en œuvre ces dernières années. Dans l'attribution des financements (et, indirectement, dans l'évaluation des chercheurs et des équipes), une place croissante est faite à des « priorités thématiques » arrêtées en fonction de considérations politiques et économiques.

La généralisation de l'évaluation en matière de recherche n'est pas nécessairement propice à l'innovation intellectuelle, l'institutionnalisation de critères d'évaluation favorisant des pratiques « prudentes ». En sciences humaines, la publication par l'Agence

\footnotetext{
7 Les caractéristiques des bénéficiaires des programmes européens de mobilité étudiante suggèrent que celle-ci n'est, dans les faits, pas ouverte à tous (Ballatore, Blöss, 2008).
} 
d'évaluation de la recherche et de l'enseignement supérieur (AERES), en 2008 et 2009, de listes de revues témoignait d'un repli disciplinaire peu compatible avec les pratiques moins routinières, mais parfois plus originales, de « l'interdisciplinarité ». Le principe de la hiérarchisation (institutionnalisée) des lieux de publications risque de privilégier les lieux les plus anciens et les plus consacrés qui, pour cette raison même, ont peu de chances d'être les plus ouverts aux travaux les plus novateurs. À cet égard, le cas de la science économique est intéressant, des critères d'évaluation des activités de publication s'y étant imposés plus tôt que dans la plupart des sciences humaines : la classification stricte des revues, la valorisation systématique des supports « internationaux » (c'est-à-dire le plus souvent états-uniens) semblent renforcer encore davantage la standardisation de la recherche autour d'une « orthodoxie » méthodologique et intellectuelle, mais aussi idéologique (Lebaron, 2000 ; Pontille,Thorny, 2008).

\section{Les implications des réformes}

Par cette dimension conservatrice, les réformes actuelles répondent au schéma ancien de l'intervention d'un pouvoir temporel dans la vie universitaire. Certes, que ces ingérences soit le fait d'un pouvoir économique, plutôt que politique ou religieux est relativement nouveau, même si le principe d'une collaboration entre l'Université et des entreprises l'est moins. En France, par exemple, avant la Seconde Guerre mondiale, des universités avaient des relations non négligeables avec l'industrie, notamment à un niveau régional (Olivier-Utard, 2003). La spécificité des réformes actuelles tient peut-être moins à l'objectif qu'elles visent, qu'à la systématicité avec laquelle elles aspirent à le mettre en œuvre, et à leur propension à intervenir dans les mécanismes mêmes de l'activité académique ; elles donnent parfois l'impression de ne pas seulement chercher à mettre cette dernière au service de l'économie, mais aussi, en quelque sorte, à l'asservir. Néanmoins, on est conduit à relativiser partiellement leur nouveauté lorsqu'on considère que les «Trente Glorieuses » auraient pu correspondre en ce domaine (comme en d'autres), non pas à un « régime normal », mais à une forme de parenthèse, entre un long XIX siècle dominé par l'essor du libéralisme économique et le retour en force opéré par ce dernier au cours des dernières décennies (Gingras, 2003). Durant cette parenthèse, la puissance publique a joué un rôle qu'elle n'endossait pas auparavant, et qui a ensuite été contesté. La demande adressée à l'enseignement supérieur et à la recherche de participer à la « modernisation économique » s'exprimait déjà (voir supra), mais pas avec la même vigueur qu'aujourd'hui, et elle était tempérée par les mots d'ordre relatifs à la démocratisation de l'accès au savoir (Bourdieu, Passeron, 1970).

Les réformes contemporaines interviennent à la suite d'une période d'après-guerre marquée par l'expansion universitaire des années 50-60 et par une transformation rapide du corps enseignant et du public de l'enseignement supérieur, dont la crise de 1968 exprima les contradictions. Peut-être plus qu'il ne peut le sembler au premier abord, la situation actuelle conserve des traces de cette crise. En effet, les forces conservatrices n'ont pas totalement oublié ces événements durant lesquels l'Université 
(re)devint un lieu d'agitation : en 2007, Nicolas Sarkozy, le candidat de droite aux élections présidentielles chercha encore dans l'invocation de ces « événements » vieux de 40 ans un instrument de mobilisation de son électorat (Ridet, 2007). La contestation des réformes n'est sans doute pas étrangère à une aspiration à prolonger un moment où, en sciences humaines notamment, les « discours critique[s] sur la société » (Mercier, 2012 : 229) ont connu une certaine prospérité.

Les réformes actuelles interviennent dans un monde académique dont certaines composantes ont des relations assez anciennes avec l'économie (qui se sont au plus distendues dans les décennies d'après-guerre) et d'autres, comme les sciences humaines, ont en quelque sorte prospéré dans l'humeur critique des années 60-70. Lorsqu'on ajoute à cela le conflit des facultés en quelque sorte séculaire, on ne peut qu'être attentif aux implications, variables selon les disciplines, des réformes en cours. Par exemple, les injonctions à s'investir, en matière de recherche, dans une vaste compétition internationale, confortent des orientations prises parfois de longue date. Dans les sciences de la nature ou dans des disciplines comme l'économie ou la psychologie, les enseignants-chercheurs ont largement intériorisé la nécessité de publier principalement (voire exclusivement) en anglais, et la loi « publish or perish » (« publier ou mourir »). \| en va autrement dans des disciplines plus littéraires ou/et moins (voire très peu) internationalisées. Dans leur cas, l'injonction implique une transformation parfois radicale des pratiques de recherche qui concerne la langue d'expression, le choix des supports de publication, mais aussi l'univers des références intellectuelles dans lequel les travaux s'inscrivent et, souvent, les objets mêmes des recherches : dans les « humanités » et les sciences humaines, quantité de sujets de recherche sont liés à des cadres locaux (et notamment nationaux) et, pour cette raison, ne seront jamais l'objet d'une compétition internationale avec des chercheurs étrangers (Gingras, Mosbah-Natanson, 20 I0).

De façon générale, les réformes impliquent des transformations qui ne peuvent avoir les mêmes conséquences dans les différentes disciplines. L'exemple de la science économique (voir supra), à laquelle le discours réformateur emprunte des justifications - c'est en effet au nom de savoirs produits par le monde académique qu'il en appelle à une transformation de cet univers -, rappelle que les réformes trouvent aussi des soutiens dans le monde académique ${ }^{8}$. En un sens, le mot d'ordre de la «professionnalisation » est constitutif de certains segments universitaires. Pour Jacques Le Goff (1999 : 199), certains pans des universités au Moyen Âge s'apparentaient déjà à des « centres de formation professionnelle ». Depuis plusieurs décennies, dans les sciences de la nature, certaines filières forment des professionnels pour les entreprises. Les choses sont plus contrastées dans les humanités et les sciences humaines. Y subsistent des disciplines dont les enseignements restent marqués par l'objectif de former de futurs enseignants (ce qui, dans l'acception aujourd'hui couramment donnée au terme, ne correspond pas à une « professionnalisation »), mais s'y sont aussi développées des filières

\footnotetext{
${ }^{8}$ Par exemple, il n'est pas anodin que, au fil du temps, les experts sollicités pour les rapports sur la question de l'évaluation des enseignants-chercheurs ont pour caractéristique d'être de plus en plus marginaux dans le monde universitaire (Garcia, 2009).
} 
interdisciplinaires organisées autour d'objets spécifiques (sciences de l'éducation, sciences de l'information et de la communication, STAPS...) et davantage orientées vers la formation de « professionnels » (Le Gall, Soulié, 2008).

De même, les disciplines sont inégalement en mesure de trouver des financements dans le secteur privé ; des partenariats public-privé existent de fait dans des filières juridiques, par exemple avec des chambres syndicales de notaires, ou dans des sciences de la nature pour des programmes de recherche appliquée (Allinne, 2000 : 4I). Ils paraissent beaucoup plus difficiles à mettre en place dans d'autres disciplines. Pour la recherche, la possibilité de collaborations avec l'industrie se concentre dans quelques domaines privilégiés, comme la chimie ou les sciences de l'ingénieur (Grossetti, Milard, 2003).

Cette diversité de l'Université doit être prise en compte dans l'interprétation des mobilisations récentes, spécifiquement concernant le mouvement autour de «Sauvons la recherche » en 2004 et la contestation, plus large, autour de la Loi relative aux libertés et responsabilités des universités (LRU) et du décret redéfinissant le statut des enseignants-chercheurs (Brisset, 2009). Ces mobilisations étaient surprenantes par leur ampleur. Mais, venus d'horizons très variés, leurs participants ne se rejoignaient peut-être que superficiellement, en particulier sur les volets des réformes consistant à étendre à l'ensemble du monde universitaire des pratiques déjà bien implantées dans certains de ses segments.

De plus, les réformes des années 2000 s'inscrivent dans des processus entamés depuis relativement longtemps. Le mouvement de professionnalisation a en quelque sorte commencé avec la création des instituts universitaires de technologie (IUT) en 1966, les maitrises de science et technique (MST) et de sciences de gestion (MSG) en 197I ou la formation continue (Denantes, 2006). Le basculement vers une « logique d'offre » pour la création de nouveaux diplômes universitaires (MignotGérard, Musselin, 200 I), la tendance à l'alourdissement des tâches administratives dans le métier d'enseignant-chercheur, le renforcement en matière de recherche (ou de « diplomation ») des exigences quantitatives de « productivité », étaient déjà bien engagés dans les années 90 . On peut penser que les transformations des structures sociales qui opèrent progressivement et s'inscrivent dans la durée tendent à produire, à la faveur du renouvellement générationnel, des agents qui y sont plus ou moins ajustés et en contestent moins les principes.

\section{Réformes et justice sociale}

Une question importante - qui ne peut être qu'effleurée ici - est le point de vue des étudiants et de leurs familles, dont les promoteurs des réformes se réclament parfois : la subordination de l'enseignement supérieur à des fins économiques répondrait au souhait des étudiants qui, s'apprêtant à entrer sur un marché du travail tendu, auraient intérêt à des réformes améliorant leur « employabilité ». 
Celles-ci seraient même indispensables à une politique de « démocratisation » conséquente de l'enseignement supérieur, la « deuxième massification » faisant entrer dans l'enseignement supérieur de nouveaux étudiants de première génération, plus exposés, par leurs caractéristiques biographiques, au chômage. Inséparablement, il faudrait considérer que « les étudiants » sont aujourd'hui plus consuméristes, plus demandeurs de savoirs pratiques. Les travaux de sociologie de la culture qui mettent en évidence que, dans leurs pratiques culturelles notamment, les jeunes générations sont moins orientés vers la culture lettrée que les précédentes (Donnat, 20 I I), renforcent cette vision des choses. Par ailleurs, une forte proportion d'étudiants (environ $50 \%$, d'après les enquêtes menées par l'Observatoire de la vie étudiante conduites au cours des années 2000) exerce aujourd'hui une activité rémunérée parallèlement aux études durant l'année universitaire ; la condition étudiante n'a plus grande chose à voir avec une « vie de bohème » coupée des réalités du marché du travail.

Ces arguments composent une vision des choses, sans doute pas totalement fausse, mais partielle. « Les étudiants » forment une population trop hétérogène pour qu'on puisse en parler comme d'une population indifférenciée qui partagerait des « vœux» et des pratiques uniformes. C'est essentiellement sur la base d'un raisonnement logique, qui les apparente à l'homo œconomicus de la théorie économique - dont, déjà dans les années 60, Pierre Bourdieu et Jean-Claude Passeron (1970) soulignait qu'elle ne pouvait rendre compte de choix d'orientation qui s'inscrivent dans des stratégies sociales des étudiants et de leurs familles beaucoup plus larges - que l'on considère comme évident leur souhait d'une professionnalisation accrue de l'enseignement supérieur. Évidemment susceptibles de produire des résultats très différents selon la formulation des questions, les sondages ne confirment d'ailleurs pas systématiquement cette croyance ${ }^{9}$. La désertion de masters « recherche » au profit de filières professionnelles n'aide pas non plus à trancher : Bernard Convert (2006) montre que, comme elle résulte manifestement en partie d'un « effet d'offre », il est très périlleux d'en conclure à un plébiscite des formations professionnalisées ou, comme on le fait parfois, à une « crise des vocations scientifiques ».

Aujourd'hui parée de toutes les vertus, la « professionnalisation », mériterait un débat (Pinto, 2009). II se pourrait que l'idéal patronal d'un ajustement entre « l'offre » de diplômés et la demande des entreprises corresponde à un objectif en fait assez peu réaliste qui engendrerait, dans la création de diplôme, des changements qui pourraient être, pour une bonne part, du seul ordre de l'affichage. Par ailleurs, la « professionnalisation » ne va pas sans inconvénient du point de vue de l'ensemble de la collectivité. D'abord, elle s'accompagne de dispositifs qui font des étudiants une main d'œuvre bon marché pour les entreprises au détriment de ceux qui recherchent du travail. Ensuite, rendre les diplômés du supérieur plus

\footnotetext{
9 L'un d'entre eux (sondage EducPros Campus communication, Ipsos, mars 2012) qui (entend) montre(r) que, durant la campagne présidentielle de 2012, « la réforme de Nicolas Sarkozy sur l'université ne convainc pas les Français », conclut que « sur l'insertion professionnelle comme sur la réussite en licence, les 15-24 ans ont une opinion plus positive que la moyenne des Français ».
} 
« employables » risque d'accroître encore le handicap, déjà considérable, dont souffre dans le monde du travail la minorité des nouvelles générations arrêtant les études au niveau du secondaire. Le discours réformateur occulte aussi les coûts que la « professionnalisation » peut avoir pour les étudiants. En effet, au mieux, elle les dote de « compétences » adaptées à l'état présent du marché du travail, mais les évolutions rapides de celui-ci peuvent faire perdre au diplôme « professionnel » une partie de sa valeur en quelques années. Cet argument peut conduire à réévaluer des formations généralistes qui, peut-être moins soucieuses de « l'employabilité » immédiate des étudiants, les arment peut-être davantage en vue de reconversions en cours de vie professionnelle (Winkin, 2003).

Si les promoteurs des réformes cherchaient prioritairement à accroître la « justice sociale », ils ne se contenteraient pas d'opposer de simples mesures symboliques (telles que les quotas à l'entrée de grandes écoles) au système de l'enseignement supérieur «à plusieurs vitesses ». Ils s'inquiéteraient aussi de ce que nombre de leurs mots d'ordre (le culte de « l'excellence », la « concentration des moyens »...) tendent à le renforcer. Les filières socialement et scolairement très sélectives de l'enseignement supérieur le deviennent de plus en plus (Albouy, Wanecq, 2003 ; Lafarge, Marchetti, 20l I). En outre, il n'est pas certain, que le principe d'une « université professionnalisée » soit aussi démocratique qu'on le dit. Intervenant à un moment où des catégories sociales jusqu'alors quasiment exclues de l'enseignement supérieur,y accèdent plus largement, la professionnalisation pourrait s'avérer, entre autres choses, un moyen de perpétuer le monopole traditionnel que les couches supérieures exercent sur cette culture lettrée qui est d'autant plus valorisée qu'elle est, en apparence du moins, « inutile ».

\section{Conclusion}

Ce texte vise à apporter quelques éléments complémentaires à l'analyse d'Arnaud Mercier (20 I 2). Certains éléments sont de nature historique, d'autres, invitent à regarder les évolutions actuelles comme inscrites dans une dynamique de transformations qui dépasse le monde académique. Un autre élément apporté consiste à engager une représentation de la communauté universitaire un peu moins idéalisée et moins indifférenciée que celle qui nous semble sous-tendre l'article d'Arnaud Mercier.

Ces éclairages modifient un peu la lecture générale des réformes : celles-ci correspondent peut-être moins à la destruction d'un idéal universitaire (qui n'a sans doute jamais été unique) qu'à une tentative d'amplifier et de généraliser à l'ensemble du monde académique des logiques qui, en un sens, y sont présentes d'assez longue date dans certains de ses segments. Notre appartenance aux sciences sociales nous porte peut-être à surestimer un peu le pouvoir de mobilisation qu'une valeur comme « l'esprit critique » peut avoir dans l'ensemble de la communauté universitaire. Simultanément, cette appartenance peut nourrir une critique des réformes qui ne consiste pas tout à fait à opposer à la conception utilitariste qui 
sous-tend les réformes l'idéal d'un savoir pur et désintéressé. Par le passé, les sciences sociales se sont souvent présentées comme plus en prise avec le monde que certaines disciplines littéraires. Elles se sont souvent voulues « utiles », souvent certes dans un sens différent de l'acception économiciste que les réformes donnent à ce mot. Ces dernières peuvent être critiquées pour leurs présupposés utilitaristes, mais aussi pour la conception rigide et restrictive qu'elles se font de « l'utilité ». Lorsqu'elles découlent de la recherche fondamentale, les « innovations » rappellent que la frontière entre un savoir « utile » et un savoir qui ne le serait pas n'existe pas. Dans un sens, l'engagement dans la recherche exclut l'idée même qu'il puisse exister des savoirs inutiles : ceux qui ne servent pas, dans l'immédiat, à améliorer la vie collective, sont au moins utiles à la connaissance, ou pourront l'être à celle de demain. C'est un coup de force, lié à l'évolution idéologique de ces dernières décennies, que d'avoir imposé l'idée que seuls sont utiles au bien-être collectif les savoirs qui peuvent produire des profits économiques à courte échéance.

L'inscription des réformes dans cette évolution très large qui prône une emprise croissante du champ économique sur l'ensemble des structures sociales attire l'attention sur quelques questions. Si, dans les années 2000, les chercheurs et les universitaires se sont mobilisés d'une façon inédite contre les réformes mises en $œ u v r e$ dans leurs secteurs professionnels, ils ne se sont pas vraiment mobilisés, du moins de façon collective, contre le développement de logiques managériales assez semblables dans d'autres domaines de la vie sociale. Leurs actions (en plus de prêter ainsi le flanc au soupçon de « corporatisme ») s'en sont peut-être trouvées limitées : si les réformes académiques relèvent d'un processus de transformations nettement plus large, est-il possible de combattre efficacement, et durablement, leurs effets dans les domaines de l'enseignement supérieur et la recherche sans chercher à désamorcer la cause générale dont elles sont le produit?

L'inscription des réformes dans un processus dépassant largement le seul monde académique peut aussi aider à rendre compte de l'un des paradoxes qu'Arnaud Mercier (2012) fait émerger : les réformes exercent des effets manifestement négatifs, y compris, parfois - comme le remarque Arnaud Mercier -, au regard de leurs objectifs affichés et font l'objet de critiques très argumentées, mais elles se poursuivent presqu'imperturbablement. S'il en est ainsi, c'est peut-être qu'elles participent d'un processus général qui, comme le suggère la réponse apportée à la crise financière depuis 2008, semble parfois ressembler à une « fuite en avant ».

\section{Références}

Albouy V.,WanecqT., 2003, « Les inégalités sociales d'accès aux grandes écoles 》, Économie et statistique, 36I, pp. 27-52.

Allinne J.-P., 2010, «Trois idées reçues sur le mécénat », pp. 17-84, in :Allinne J.-P., Carrier R., éds, La Culture au risque du marché : le mécénat face à ses acteurs, Paris, Éd. L'Harmattan. 
Ballatore M., Blöss T., 2008, « L'autre réalité du programme Erasmus : affinité sélective entre établissements et reproduction sociale des étudiants », Formation Emploi, I03, pp. 57-74.

Bourdieu P., 1984, Homo Academicus, Paris, Éd. de Minuit.

— 1989, La Noblesse d'État. Grandes écoles et esprit de corps, Paris, Éd. de Minuit.

Bourdieu P., Boltanski L., 1975, « Le titre et le poste :rapports entre le système de production et le système de reproduction », Actes de la recherche en sciences sociales, 2, pp. 95- 107.

Bourdieu P., Passeron J.-Cl., 1970, La Reproduction. Éléments pour une théorie du système d'enseignement, Paris, Éd. de Minuit.

Brisset C.-A., éd., 2009, L'université et la recherche en colère. Un mouvement social inédit, Bellecombe-en-Bauges, Éd. Le Croquant.

Charle C., 1994, La République des universitaires. 1870-1940, Paris, Éd. Le Seuil.

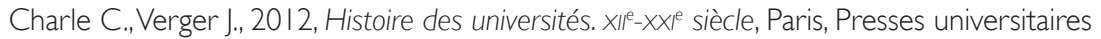
de France.

Convert B., 2006, Les impasses de la démocratisation scolaire. Sur une prétendue crise des vocations scientifiques, Paris, Éd. Raisons d'agir.

Denantes J., 2006, Les universités françaises et la formation continue. 1968-2002, Paris, Éd. L'Harmattan.

Donnat O., 2011, « Pratiques culturelles, 1973-2008. Dynamiques générationnelles et pesanteurs sociales », Culture Études, 7, pp. I-35.

Durkheim É., 1938, L'évolution pédagogique en France, Paris, Presses universitaires de France, 1990.

Duval J., 2007, Le Mythe du « trou de la Sécu », Paris, Éd. Raisons d'agir.

Garcia S., 2008, « L'expert et le profane : qui est juge de la qualité universitaire », Genèses, 70, pp. 66-87.

— 2009, «Réformes de Bologne et économicisation de l'enseignement s supérieur », Revue du Mauss, 33, pp. I54- 172.

Gibbons M., Limoges C., Nowony H. et al., 1994, The New Production of Knowledge. The Dynamics of Science and Research in Contemporary Societies, Los Angeles/Londres, Éd. Sage.

Gingras Y., « Idées d'universités », Actes de la recherche en sciences sociales, I48, pp. 3-7.

Gingras Y., Mosbah-Natanson S., 20 I 0, « La question de la traduction en sciences sociales : les revues françaises entre visibilité internationale et ancrage national ». Accès : http:// www.cirst.uqam.ca/Portals/0/docs/note_rech/20 l 0-02.pdf. Consulté le 25/05/I3.

Grossetti M., Milard B., 2003, « Les évolutions du champ scientifique en France à travers les publications et les contrats de recherche », Actes de la recherche en sciences sociales, I48, pp. 47-56.

Karpik L., 20 I2, «"Performance", "excellence" et création scientifique », Revue française de socio-économie, 10, pp. II3-135.

Lafarge G., Marchetti D., 20 I I, « Les portes fermées du journalisme », Actes de la recherche en sciences sociales, 189, pp. 72-99.

Lebaron F., 2000, La croyance économique. Les économistes entre science et politique, Paris, Éd. Le Seuil. 
Lenoir R., 1989, « Objet sociologique et problème social », pp. 53- 100, in : Champagne P. et al., Initiation à la pratique sociologique, Paris, Dunod.

Le Gall B., Soulié C., 2008, « Massification, professionnalisation, réforme du gouvernement des universités et actualisation du conflit des facultés », pp. 173-208, in: ARESER (Association de réflexion sur les enseignements supérieurs et la recheche), 2007, Les ravages de la " modernisation » universitaire en Europe, Paris, Éd. Syllepse.

Le Goff J., 1999, Un autre Moyen Âge, Paris, Gallimard.

Malissard P., Gingras Y., Gemme B., 2003, « La commercialisation de la recherche », Actes de la recherche en sciences sociales, 148, pp. 57-67.

Mignot-Gérard S., Musselin C., 200I, « L'offre de formation universitaire : à la recherche de nouvelles régulations », Éducation et sociétés, 8, pp. I I-25.

MEN (ministère de l'Éducation nationale), 2012, Repères et références statistiques - édition 2012, Paris, ministère de l'Éducation nationale.

Mercier A., 2012, «Dérives des universités, périls des universitaires », Questions de communication, 22, pp. 197-234.

MESR (ministère de l'Enseignement supérieur et de la Recherche), L'État de l'enseignement supérieur et de la recherche en France, 5.

Montlibert Chr. de, 2004, Savoir à vendre, Paris, Éd. Raisons d'agir.

Musselin C., 2008, «Vers un marché international de l'enseignement supérieur? », Critique internationale, 39, pp. 13-24.

Olivier-Utard F., 2003, « La dynamique d'un double héritage », Actes de la recherche en sciences sociales, I48, pp. 20-33.

Parisot L., Pébereau M., 20 I2, « Pour une école exigeante, personnalisée et créative », Le Figaro, 26 oct., p. 14.

Pinto V., 2008, «"Démocratisation" et "professionnalisation" de l'enseignement supérieur 》, Mouvements, 55, pp. $12-23$.

Polanyi K., 1944, La grande transformation. Aux origines politiques et économiques de notre temps, trad. de l'anglais par C. Malamoud, Paris, Gallimard, 1983.

Pontille D., Torny D., 2010, « Revues qui comptent, revues qu'on compte : produire des classements en économie et gestion », Revue de la régulation, 8 . Accès : http://regulation. revues.org/888।. Consulté le 25/05/I3.

Ridet Ph., 2007, « M. Sarkozy transforme son meeting parisien en démonstration de forece et de popularité », Le Monde, 2 mai, p. 9.

Ropé F., Tanguy L., éds, 1994, Savoirs et compétences. De l'usage de ces notions dans l'école et l'entreprise, Paris, Éd. L'Harmattan.

Spector M., Kitsuse J., 2009, Constructing Social Problems, New Brunswick/Londres, Transaction Publishers.

Verger J., 1973, Les Universités au Moyen Âge, Paris, Presses universitaires de France.

Winkin Y., 2003, La Communication n'est pas une marchandise. Résister à l'agenda de Bologne, Bruxelles, Éd. Labor. 